\title{
Adult mesenchymal stem cells for the treatment in patients with rotator cuff disease: present and future direction
}

\author{
Dong Rak Kwon, Gi-Young Park \\ Department of Rehabilitation Medicine, Catholic University of Daegu School of Medicine, Daegu, South Korea \\ Correspondence to: Dong Rak Kwon, MD, PhD. Department of Rehabilitation Medicine, Catholic University of Daegu School of Medicine, 33 \\ Duryugongwon-ro 17-gil, Nam-Gu, Daegu, 42472, South Korea. Email: coolkwon@cu.ac.kr; dongrakkwon@hotmail.com. \\ Provenance: This is an invited Editorial commissioned by Section Editor Liuhua Zhou (Department of Urology, Nanjing First Hospital, Nanjing \\ Medical University, Nanjing, China). \\ Comment on: Jo CH, Chai JW, Jeong EC, et al. Intratendinous injection of autologous adipose tissue-derived mesenchymal stem cells for the \\ treatment of rotator cuff disease: a first-in-human trial. Stem Cells 2018;36:1441-50.
}

Submitted Aug 20, 2018. Accepted for publication Sep 07, 2018.

doi: $10.21037 / \mathrm{atm} .2018 .09 .06$

View this article at: http://dx.doi.org/10.21037/atm.2018.09.06

Rotator cuff tendon tear (RCTT) is reported to be one of the most common causes of chronic shoulder pain in the United States with more than 250,000 RCTT repairs each year (1). Individuals under 60 years of age show a $6 \%$ incidence of full-thickness RCTT, while those over 60 show an incidence of $30 \%$, as determined by cadaveric studies (2). RCTT results in considerable disability and diminished quality of life for many patients (3).

Chronic tendon degeneration is currently considered a major contributor to the pathology of RCTT (4). Treatment for RCTT includes conservative treatments such as exercise, electrotherapy, acupuncture, manual treatment, taping, steroid and platelet-rich plasma injections and surgical treatment (5). Surgical repair for tendon tear is one of the most common orthopedic procedures undertaken today (6).

The classification of RCTT using a reliable classification method is critical to improve the treatment of rotator cuff disease. This classification has been used to determine the appropriate treatments to achieve the best results for each type of tendon tear. Many factors such as tear size, shape, retraction, and fatty infiltration have been identified as important factors when assessing a patient with a symptomatic RCTT. RCTT are classified as either partialor full-thickness. Partial-thickness tears are focal defects in the rotator cuff tendon and involve only bursal or articular surface or intratendinous. Full-thickness tears extend from the articular surface to the bursal surface of the tendon.

Ellman (7) proposed a classification system for partial- thickness RCTT based on intra-operative findings according to the location (bursal-side, articular-side, and intratendinous), grade, and tear area $\left(\mathrm{mm}^{2}\right)$. In his classification, a grade 3 tear is $>6 \mathrm{~mm}$ deep $(>50 \%)$, a grade 2 tear is $3-6 \mathrm{~mm}$ in depth but not exceeding one-half of the tendon thickness (25-50\%), and a grade 1 tear is considered as $<3 \mathrm{~mm}$ deep ( $<25 \%$ ). DeOrio and Cofield (8) developed classification systems for full-thickness RCTT, which involved measuring the anterior-posterior length of the tendon that was torn off of the humeral head at the time of surgery. Their system classified tendon tear size as massive if the tear was greater than $5 \mathrm{~cm}$, large if the tear was $3-5 \mathrm{~cm}$, medium if the tear was $1-3 \mathrm{~cm}$, and small if the tear was less than $1 \mathrm{~cm}$ in length.

For acute tears, and in patients younger than 65 years, in the case of chronic, reparable tears larger than $1 \mathrm{~cm}$ and without significant chronic muscle changes (such as atrophy, fatty infiltration, and impairment of the biomechanical properties of the repair sites), early surgical repair can be considered. Early surgical repair can also be considered in large bursal-sided partial thickness tears (larger than $6 \mathrm{~mm}$ or more than $50 \%$ of the tendon thickness) because the risk of tear progression and rate of failure of conservative treatment is high (9).

However, the failure rate for rotator cuff tendon repair is $20-90 \%$ (10) and the outcome can vary depending on the patient's age, tear size, chronicity, muscle atrophy, fatty degeneration, tendon quality, operative technique, and postoperative rehabilitation treatment (11). Revision rotator 
cuff surgery has been less successful than primary repair (12).

Current therapeutic approaches result in suboptimal quality at the distal end of the RCTT and a lack of physiological restoration; thus, a new biological treatment, which creates an optimized environment for the restoration of the tendon, is needed to regenerate the tendon and reduce scar formation (13).

The paper being referenced in this editorial [published in the Stem Cells on May 11, 2018 (14)] shows that injecting autologous adipose tissue-derived mesenchymal stem cells (ADMSCs) into the tendon without surgery for the treatment of rotator cuff disease is feasible, safe, and effective to regenerate of tendon defect being seen.

Tissue engineering approaches are now able to target and generate almost any cell of the body. Stem-cell-based research has grown exponentially over the past few years, drawing much attention from scientific and public, with various types of stem cells being considered for clinical applications. Embryonic stem cells and induced pluripotent stem cells have nearly infinite potential to differentiate in vitro and in vivo into specific progenitor cells and into mature and specialized cell lineages of all 3 embryonic germ layers and are very promising their (15). However, the clinical use of these cells is limited by scientific and clinical issues of safety, such as teratoma formation (16).

Mesenchymal stem cells (MSCs) are a very attractive source of cells for several reasons for clinical treatment. MSCs can be isolated with relative ease from multiple sources, including the bone marrow (BM), adipose tissue (AT), and umbilical cord blood (UCB). MSCs can self-renew for several passages before reaching a state of senescence. They have been used to treat immune diseases because of their inherent immunomodulatory characteristics. MSCs have been shown to produce anti-inflammatory cytokines and suppress the proliferation, differentiation, and function of immune cells in vitro (17). In vivo, transplanted MSCs do not elicit an immune response, allowing their use in allogenic stem cell therapy $(18,19)$. MSCs can be induced to directly differentiate into musculoskeletal cell types. They do not form teratomas when implanted in vivo, making them safe for use in clinical applications (20).

The BM was the first reported source of MSCs. Although BM remains the major source of multipotent MSCs, harvesting is a very invasive procedure. In addition, the number, the potential for differentiation, and the maximum life span of BM-MSCs decrease with increasing age (21). Therefore, intensive investigation is underway to isolate alternative sources of MSCs.
One such alternative source is AT, which can be obtained by a less invasive method and in larger quantities than BM. AT contains stem cells similar to BM-MSCs, called processed lipoaspirate (PLA) cells (22). These cells can be separated from a large number of cosmetic liposuctions, easily grown under standard tissue culture conditions, and show multi-lineage differentiation capacity (22).

UCB is another alternative source that can be obtained by a minimally invasive method without harming the mother or the baby (23). UCB-MSCs have the low frequency, however it has the highest expansion potential among all the cell sources. Considering the clinical applications, the resulting cell numbers may be similar to both $\mathrm{BM}$ and $\mathrm{AT}$ with higher frequencies.

Of the various MSCs, human UCB-MSCs have the greatest therapeutic potential due to various attributes, such as the ability to home in on injured tissue, low immunogenicity, multi-directional differentiation, and extensive secretion profiles (23). Allogenic UCB-MSCs can be particularly beneficial to elderly patients, or ones with significant comorbidities, in whom the function of autologous MSCs is impaired (24). In addition, UCB-MSCs can be produced commercially in larger quantities without compromising on quality.

The study by Jo et al. (14) was performed to assess the safety and feasibility of autologous ADMSCs for the treatment of rotator cuff disease. Intratendinous administration of a single dose of ADMSCs of up to $1.0 \times 10^{8}$ cells $/ 3 \mathrm{~mL}$ of saline was well tolerated in 19 patients with RCTT, with no evidence of ADMSCs injection-associated serious or serious adverse events at any of the three doses tested. The most common adverse event during the study period was back pain in three patients. Back pain was considered to be unrelated to the ADMSCs by the clinical investigators and was managed with appropriate treatment. Moreover, there was also a follow-up scheduled for up to 6 months after ADMSCs injection to confirm the long-term safety of the treatment. As far as we know, this study documents the first clinical trial of ADMSCs for the treatment of rotator cuff disease. The results showed significant improvement in SPADI scores, shoulder pain, MRI examination, and arthroscopic examination in the midand high-dose groups.

Before application of MSCs can be considered, it should be clarified whether there is a critical optimum dose (including repeated doses) of cells for RCTT for safety and therapeutic benefit (25). It is reasonable that the optimal number of cells might vary depending on the size of the 
rotator cuff tendon defect, the cell type used, and the particular region in need of regeneration. The dose-efficacy relationship has not been well studied. Only one study so far has assessed the dose-dependent response to allogeneic ADMSCs used to treat lateral epicondylosis in humans, with participants receiving either $10^{6}$ cells or $10^{7}$ cells (26). The authors of the study reported faster pain improvement and earlier plateauing of performance scores in the group that received the higher dose, but there were no significant differences in elbow pain and performance changes between the 2 groups at any of the follow-ups. However, too small participants can make it difficult to conclude that the dosedependent response to the MSCs was significant. In the study by Jo et al. (14), the authors established an optimum ADMSCs dose that would show regenerative effects in human patients with rotator cuff disease.

Choosing an effective delivery method in MSCs therapies is a crucial step. The two main methods of delivery are direct injection of a cell suspension alone and implantation of cells that are placed in a matrix carrier vehicle. Direct delivery of MSCs seems to be a more plausible approach for tendons. In their study, Jo et al. (14) injected ADMSCs through the lateral aspect of the shoulder under ultrasonographic guidance. In previous studies, one-third of the blind shoulder injections, performed by experienced physician, were shown to be inaccurate (27).

Polydeoxyribonucleotide (PDRN) is a biologic adjuvant that has the same advantages as UCB-MSCs in terms of commercial mass production. PDRN is a mixture of DNA polymers with chain lengths ranging from 50 to 2,000 bp. It is extracted from trout sperm and purified as a preparation containing a high percentage of DNA. PDRN is a source of pyrimidines and purines, and stimulates nucleic acid synthesis through the salvage pathway (28). PDRN can induce angiogenesis and collagen synthesis and exhibits anti-inflammatory activity (29). Recently, an animal study (18) showed using histological and motion analysis that co-injection of UCB-MSCs and PDRN was more effective than UCB-MSCs injection alone in a rabbit model of chronic traumatic full-thickness RCTT. One published clinical study reported the effectiveness of PDRN in the treatment of chronic rotator cuff disease (30). Therefore, PDRN is a candidate that can enhance the effectiveness of MSCs.

In this study, the shoulder into which the ADMSCs were injected intratendinously was immobilized for 4 weeks using an abduction brace. Deconditioning and reduced muscle activity is estimated to be the most important factors leading to muscle atrophy and loss of function in patients (31). In particular, the treatment of soft tissue and bone injuries by immobilization may result in skeletal muscle atrophy and decreased functional performance (32).

Microcurrent therapy (MT) involves the therapeutic application of very low electric current (less than $1 \mathrm{~mA}$ ), which is usually sub-sensory to the body. One study demonstrated that MT may prevent progression of GCM muscle atrophy and facilitate the regeneration of muscle cells (32). These effects may increase protein synthesis and stimulate satellite cell proliferation. Therefore, MT has the potential to become an effective therapeutic intervention for the recovery of muscle atrophy due to immobilization.

The intensity of MT plays an important role in the effective treatment of muscle damage. Studies have shown that when an electric current with an intensity of less than $1,000 \mu \mathrm{A}$ was applied for treating muscle damage which increases the adenosine triphosphate (ATP) production in the tissue and stimulates the amino acids incorporated into the protein in the tissue by $30-40 \%$ above the control level along with the activation of myogenic precursor cells (satellite cells) $(32,33)$. On the other hand, when the intensity exceeded $1,000 \mu \mathrm{A}$, these bio-stimulatory effects were reversed. One study using $25 \mu \mathrm{A}$ to treat muscle atrophy reported good regeneration in vivo and in vitro (32). Therefore, it is suggested that a combination of microcurrent treatments may be helpful to prevent muscle atrophy caused by immobilization.

The role of imaging study in the patients with RCTT is to guide treatment decisions whether the patients will be managed conservatively or surgically. Various imaging methods have been used to evaluate the RCTT including arthrography, ultrasound, sonoelastography, arthrosonography, MRI, and MR arthrography. Singlecontrast arthrography of the shoulder is a valuable diagnostic method and can produce a high accuracy for the detection of full-thickness tendon tears of the rotator cuff. It can be helpful in assessing deep-surface, incomplete tendon tears of the rotator cuff but may fail to detect bursalside partial-thickness tendon tears (34).

Jo et al. (14) conducted serial arthroscopy to evaluate the gross findings but not the mechanical properties of regenerated RCTT.

Sonoelastography is a recently developed technique based on ultrasound that assesses tissue elasticity in real-time. It is based on the principle that pressure acting on tissue induces changes in the tissue depending on its elastic properties and tissue compression produces strain (displacement), 

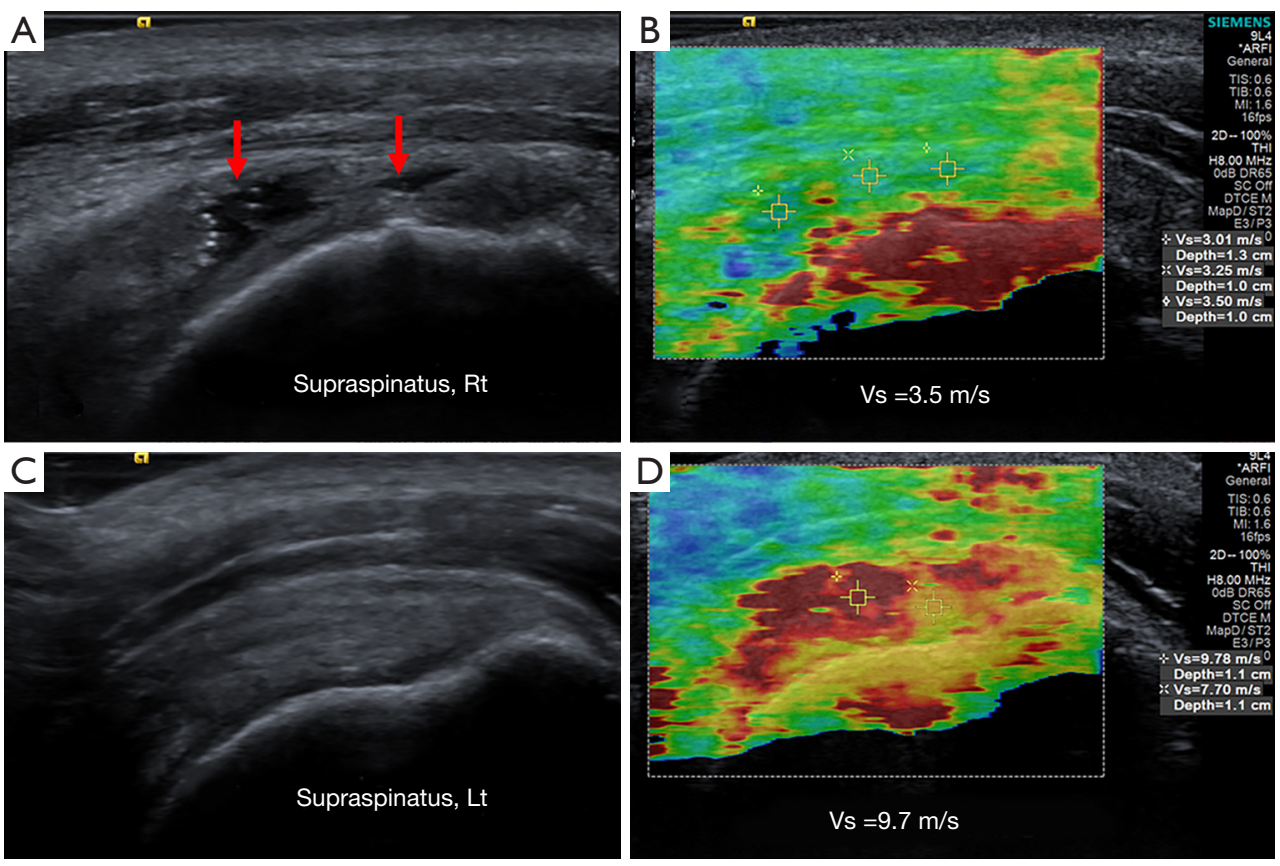

Figure 1 B-mode ultrasonography and shear wave sonoelastography using acoustic radiation force impulse imaging of supraspinatus tendon in patient with right shoulder pain. (A) Longitudinal ultrasonography of the right shoulder showed full-thickness supraspinatus tendon tear (red arrows) and (B) the highest shear wave velocity of torn supraspinatus tendon in a selected region of interest (ROI) was $3.5 \mathrm{~m} / \mathrm{sec}$; (C) longitudinal ultrasonography of the left shoulder showed intact supraspinatus tendon and (D) the highest shear wave velocity of intact supraspinatus tendon in ROI was $9.7 \mathrm{~m} / \mathrm{sec}$. Shear wave velocity of the intact supraspinatus tendon was higher than that in full-thickness supraspinatus tendon tear. The slower velocity indicates less stiffness.

which is higher in soft tissue and lower in hard tissue (35). This technique has numerous advantages including its low cost, short examination time, noninvasiveness, and simple clinical accessibility compared to using magnetic resonance elastography to estimate tissue elasticity. Shear wave sonoelastography using acoustic radiation force impulse imaging technology measure shear wave velocity that calculate the mechanical stiffness property of tissue objectively and directly (Figure 1). Sonoelastography may be used to assess the mechanical properties of rotator cuff tendon under clinical conditions and may be more effective than ultrasound and MRI in detecting subclinical tendon disorder (36).

Therefore, sonoelastography can be applied for the early diagnostics and monitoring of treatment by assessing biochemical and pathophysiological changes in the course of RCTT (37).

Arthrosonography involves a combination of ultrasound and single-contrast arthrography and provides more information about the size of RCTT and synovial proliferation in the long biceps tendon sheath or subacromial bursa for treatment planning (38). Additionally, it is an effective imaging method for assessing the postoperative integrity of the repaired rotator cuff tendon and appears to be an appropriate imaging method for replacing ultrasound (39).

MR arthrography is more sensitive and specific than either MRI or ultrasound in the diagnosis of both partialand full-thickness RCTT. Therefore, MR arthrography can be used when ultrasound and MRI diagnosis do not provide definitive results (40).

Jo et al. (14) concluded that intratendinous injection of autologous ADMSCs for the treatment of rotator cuff disease is feasible, safe, and effective and provide evidence of regeneration of tendon defect without surgery. However, they did not provide any data on the size of rotator cuff tear, which is important for determining the treatment method.

In conclusion, this study provides a foundation for future stem cell-based treatment approaches in humans, which can be used to promote the clinical application of various therapies combining stem cells and chemical agents and/or physical methods to facilitate recovery from RCT. 
Additionally, the use of sonoelastography, an imaging method that can assess the quality of the regenerated rotator cuff, may further aid clinical management.

\section{Acknowledgements}

Funding: This research was supported by Basic Science Research Program through the National Research Foundation of Korea (NRF) funded by the Ministry of Education (NRF-2016R1D1A1B01014260).

\section{Footnote}

Conflicts of Interest: The authors have no conflicts of interest to declare.

\section{References}

1. Vitale MA, Vitale MG, Zivin JG, et al. Rotator cuff repair: an analysis of utility scores and cost-effectiveness. J Shoulder Elbow Surg 2007;16:181-7.

2. Lehman C, Cuomo F, Kummer FJ, et al. The incidence of full thickness rotator cuff tears in a large cadaveric population. Bull Hosp Jt Dis 1995;54:30-1.

3. Feeley BT, Gallo RA, Craig EV. Cuff tear arthropathy: current trends in diagnosis and surgical management. J Shoulder Elbow Surg 2009;18:484-94.

4. Baydar M, Akalin E, El O, et al. The efficacy of conservative treatment in patients with full-thickness rotator cuff tears. Rheumatol Int 2009;29:623-8.

5. Berger MJ, Adams SD, Tigges BM, et al. Differentiation of umbilical cord blood-derived multilineage progenitor cells into respiratory epithelial cells. Cytotherapy 2006;8:480-7.

6. Boiani M, Schöler HR. Regulatory networks in embryoderived pluripotent stem cells. Nat Rev Mol Cell Biol2005;6:872-84.

7. Ellman H. Diagnosis and treatment of incomplete rotator cuff tears. Clin Orthop Relat Res 1990;254:64-74.

8. DeOrio JK, Cofield RH. Results of a second attempt at surgical repair of a failed initial rotator cuff repair. J Bone Joint Surg Am 1984:66:563-7.

9. Tashjian RZ. Epidemiology, natural history, and indications for treatment of rotator cuff tears. Clin Sports Med 2012;31:589-604.

10. Castricini R, Longo UG, De Benedetto M, et al. Plateletrich plasma augmentation for arthroscopic rotator cuff repair: a randomized controlled trial. Am J Sports Med 2011;39:258-65.
11. Chang YS, Choi SJ, Sung DK, et al. Intratracheal transplantation of human umbilical cord blood derived mesenchymal stem cells dose-dependently attenuates hyperoxia-induced lung injury in neonatal rats. Cell Transplant 2011;20:1843-54.

12. Chang YS, Oh W, Choi SJ, et al. Human umbilical cord blood-derived mesenchymal stem cells attenuate hyperoxia-induced lung injury in neonatal rats. Cell Transplant 2009;18:869-86.

13. Cheung EV, Silverio L, Sperling JW. Strategies in biologic augmentation of rotator cuff repair: a review. Clin Orthop Relat Res 2010;468:1476-84.

14. Jo CH, Chai JW, Jeong EC, et al. Intratendinous Injection of Autologous Adipose Tissue-Derived Mesenchymal Stem Cells for the Treatment of Rotator Cuff Disease: A First-In-Human Trial. Stem Cells 2018;36:1441-50.

15. Trounson A. The production and directed differentiation of human embryonic stem cells. Endocr Rev 2006;27:208-19.

16. Thomson JA, Itskovitz-Eldor J, Shapiro SS, et al. Embryonic stem cell lines derived from human blastocysts. Science 1998;282:1145-7.

17. Trivedi P, Hematti P. Derivation and immunological characterization of mesenchymal stromal cells from human embryonic stem cells. Exp Hematol 2008;36:350-9.

18. Kwon DR, Park GY, Lee SC. Treatment of FullThickness Rotator Cuff Tendon Tear Using Umbilical Cord Blood-Derived Mesenchymal Stem Cells and Polydeoxyribonucleotides in a Rabbit Model. Stem Cells Int 2018;2018:7146384.

19. Park GY, Kwon DR, Lee SC. Regeneration of FullThickness Rotator Cuff Tendon Tear After UltrasoundGuided Injection With Umbilical Cord Blood-Derived Mesenchymal Stem Cells in a Rabbit Model. Stem Cells Transl Med 2015;4:1344-51.

20. Krizhanovsky V, Lowe SW. Stem cells: The promises and perils of p53. Nature 2009;460:1085-6.

21. Mueller SM, Glowacki J. Age-related decline in the osteogenic potential of human bone marrow cells cultured in three-dimensional collagen sponges. J Cell Biochem 2001;82:583-90.

22. Zuk PA, Zhu M, Ashjian P, et al. Human adipose tissue is a source of multipotent stem cells. Mol Biol Cell 2002;13:4279-95.

23. Bai $\mathrm{L}, \mathrm{Li} \mathrm{D}, \mathrm{Li}$ J, et al. Bioactive molecules derived from umbilical cord mesenchymal stem cells. Acta Histochem 2016;118:761-9.

24. Kasper G, Mao L, Geissler S, et al.Insights into 
mesenchymal stem cell aging: involvement of antioxidant defense and actin cytoskeleton. Stem Cells 2009;27:1288-97.

25. Docheva D, Müller SA, Majewski M, et al. Biologics for tendon repair. Adv Drug Deliv Rev 2015;84:222-39.

26. Lee SY, Kim W, Lim C, et al. Treatment of lateral epicondylosis by using allogeneic adipose-derived mesenchymal stem cells: apilot study. Stem Cells 2015;33:2995-3005.

27. Mattie R, Kennedy DJ. Importance of Image Guidance in Glenohumeral Joint Injections: Comparing Rates of Needle Accuracy Based on Approach and Physician Level of Training. Am J Phys Med Rehabil 2016;95:57-61.

28. Galeano M, Bitto A, Altavilla D, et al. Polydeoxyribonucleotide stimulates angiogenesis and wound healing in the genetically diabetic mouse. Wound Repair Regen 2008;16:208-17.

29. Kim JK, Chung JY. Effectiveness of polydeoxyribonucleotide injection versus normal saline injection for treatment of chronic plantar fasciitis: a prospective randomised clinical trial. Int Orthop 2015;39:1329-34.

30. Yoon YC, Lee DH, Lee MY, et al. Polydeoxyribonucleotide Injection in the Treatment of Chronic Supraspinatus Tendinopathy: A Case-Controlled, Retrospective, Comparative Study With 6-Month Follow-Up. Arch Phys Med Rehabil 2017;98:874-80.

31. Barreiro E, Bustamante V, Cejudo P, et al. Guidelines for the evaluation and treatment of muscle dysfunction in patients with chronic obstructive pulmonary disease. Arch Bronconeumol 2015;51:384-95.

32. Moon YS, Kwon DR, Lee YJ. Therapeutic effect of

Cite this article as: Kwon DR, Park GY. Adult mesenchymal stem cells for the treatment in patients with rotator cuff disease: present and future direction. Ann Transl Med 2018;6(22):432. doi: 10.21037/atm.2018.09.06 microcurrent on calf muscle atrophy in immobilised rabbit. Muscle Nerve 2018;58:270-6.

33. Cheng N, Van Hoof H, Bockx E, et al. The effects of electric currents on ATP generation, protein synthesis, and membrane transport of rat skin. Clin Orthop Relat Res 1982;171:264-72.

34. Neviaser RJ. Radiologic assessment of the shoulder. Plain and arthrographic. Orthop Clin North Am 1987;18:343-9.

35. Park GY, Kwon DR. Application of real-time sonoelastography in musculoskeletal diseases related to physical medicine and rehabilitation: an invited review. Am J Phys Med Rehabil 2011;90:875-86.

36. Park G, Kwon D, Park J. Diagnostic confidence of sonoelastography as adjunct to greyscale ultrasonography in lateral elbow tendinopathy. Chin Med J 2014;127:3110-5.

37. Seo JB, Yoo JS, Ryu JW. Sonoelastography findings of supraspinatus tendon in rotator cuff tendinopathy without tear: comparison with magnetic resonance images and conventional ultrasonography. J Ultrasound 2014;18:143-9.

38. Lee HS, Joo KB, Park CK, et al. Sonography of the shoulder after arthrography (arthrosonography): preliminary results. J Clin Ultrasound 2002;30:23-32.

39. Lee KW, Yang DS, Chun TJ, et al. A comparison of conventional ultrasonography and arthrosonography in the assessment of cuff integrity after rotator cuff repair. Clin Orthop Surg 2014;6:336-42.

40. de Jesus JO, Parker L, Frangos AJ, et al. Accuracy of MRI, MR arthrography, and ultrasound in the diagnosis of rotator cuff tears: a meta-analysis. AJR Am J Roentgenol 2009;192:1701-7. 